Title:

Carbon Nanoparticle-Reinforced Metal Matrix Composites: Microstructural Tailoring and Predictive Modeling

\title{
Authors:
}

Leander Reinert, Sebastián Suarez, Thomas Müller, Frank Mücklich

This is the peer reviewed version of the following article:

Reinert, L., Suarez, S., Müller, T. and Mücklich, F. (2017), Carbon Nanoparticle-Reinforced Metal Matrix Composites: Microstructural Tailoring and Predictive Modeling . Adv. Eng. Mater., 19: 1600750.

doi:10.1002/adem.201600750,

which has been published in final form at https://doi.org/10.1002/adem.201600750. This article may be used for non-commercial purposes in accordance with Wiley Terms and Conditions for Use of Self- Archived Versions. 
DOI: 10.1002/adem.201600750

\section{Carbon nanoparticle-reinforced metal matrix composites: Microstructural tailoring and predictive modelling.}

By Leander Reinert, Sebastian Suarez*, Thomas Müller, and Frank Mücklich

[*] Dipl.-Ing. L. Reinert, Dr.-Ing. S. Suarez (Corresponding-Author), Thomas Müller, Prof. Dr. F. Mücklich

Chair of Functional Materials, Dept. of Materials Science and Engineering, Saarland University, Campus D3 3, Saarbrücken D-66123, Germany.

E-mail: s.suarez@mx.uni-saarland.de

[**] Acknowledgements: The present work is supported by funding from the Deutsche Forschungsgemeinschaft (DFG, project: MU959/38-1 and project: SU 911/1-1). The authors wish to acknowledge the EFRE Funds of the European Commission for support of activities within the AMELab project. This work was supported by the CREATe-Network Project, Horizon 2020 of the European Commission (RISE Project No. 644013). The authors would like to thank Prof. Dr. Volker Presser (INM, Saarbrücken) for providing the OLC and $\mathrm{nD}$ powder.

\section{Abstract}

Nickel matrix composites are produced with concentrations of $0.5-10$ vol. $\%$ of carbon nanotubes (MWCNTS), onion-like carbon (OLC) or nanodiamonds $(n D s)$ as reinforcements by hot pressing. After densification, a secondary annealing step is conducted to induce grain growth and thus to analyse the effect of the different carbon nanoparticles $(C N P)$ and their concentrations on the microstructure. Grain sizes are measured by electron backscatter diffraction and a model based on the Zener equation is adapted to predict the observed grain refinement for all CNP. It is shown that the model is valid, as long as no saturation value of the refinement effect is reached. However, the individual concentration at which a saturation value is reached differs, which is correlated to the mean CNP agglomerate diameter distribution. For MWCNTs and OLCs, an increasing grain refinement is observed for up to 3 vol.\% and 6.5 vol.\%, respectively. However, for $n D$ s the mean grain size is decreasing up to 10 vol.\%. This difference is correlated to different hybridization states or different particle geometries for all CNP. As information regarding hybridization state and particle 
morphology of CNP is considered in this study, it provides valuable information for CNPreinforced MMC to a general extent.

\section{Introduction}

Composite materials provide the capability of tailoring their properties (mechanical, electrical, thermal, etc.) by managing simple variables such as the chosen reinforcement material, the reinforcement volume fraction or the processing parameters. For those reasons, they have been in the spotlight of materials research for the last few decades. At the same time, carbon nanomaterials have been foreseen as very promising candidates as reinforcement phase due to their outstanding intrinsic properties and low density ${ }^{[1-3]}$, which are essential features for composite materials. When used in composites, they can lead to increased strength ${ }^{[4-6]}$, hardness ${ }^{[7-9]}$, electrical and thermal conductance ${ }^{[8,10,11]}$, thermomechanical stability ${ }^{[12]}$ as well as a reduction in friction and wear ${ }^{[13-15]}$, just to enumerate some examples. In general, carbon nanomaterials can be found in many different morphologies and configurations. Specifically for this work, the focus is placed on three types of carbon nanoparticles (CNP), namely: carbon nanotubes $(\mathrm{CNT})^{[16]}$, nanodiamonds $(\mathrm{nD})^{[3]}$, and onion-like carbons (OLC) ${ }^{[17]}$. These CNP were selected as they show either a specific carbon hybridization or particle geometry, therefore covering a wide span of carbon nanomaterials and allowing a systematic analysis of the effect of those differences on the microstructure in a metal matrix composite (MMC). One of the advantages would be that all those CNP possess a certain degree of chemical inertness and behave in the same way when no interphases are formed with the matrix in MMC (e.g. $\mathrm{Cu}, \mathrm{Ag}$, etc.).

For CNP-reinforced metal matrix composites, the material strengthening due to grain refinement has been identified in several different matrices ${ }^{[8,18-21]}$. In general, the magnitude of the reinforcing effect generated by CNP in a metal matrix composite is strongly affected by their distribution ${ }^{[5,22,23]}$ which directly influences the mechanical properties of the material 
(e.g., hardness or strength) due to grain boundary strengthening ${ }^{[21]}$. Also, rather small agglomerates are known to enable the Orowan mechanism thus leading to a reinforcing effect of the composite ${ }^{[24,25]}$. Furthermore, a finer distribution of small agglomerates creates a larger amount of interface between reinforcement and matrix, efficiently increasing the load transfer during stress ${ }^{[5]}$. Additionally, other factors such as the generation of dislocations resulting from thermal mismatch between matrix and reinforcements can also contribute to an expected strengthening of the material ${ }^{[26]}$.

In this context, the main drawback of working with CNP is their tendency to form agglomerates due to intermolecular forces like dipole-dipole forces, van der Waals forces ${ }^{[27-}$ ${ }^{29]}$ or as a consequence of the synthesis methods themselves ${ }^{[30-33]}$. In a previous study, an alternative processing route has been proposed ${ }^{[23]}$, which allows for the direct comparison of the aforementioned three CNP in terms of their distribution and the generated microstructure when used as reinforcement phase in a nickel metal matrix composite. Nickel appears to be a suitable candidate as matrix material for a comparative study, since it only forms metastable carbides under very specific conditions, as shown in a previous study reported by Suarez et al. ${ }^{[21]}$. Considering the possibility of a prediction of the resulting mean grain size of a biphasic material as a function of the amount of the secondary phase (that with lower volume fraction), Smith ${ }^{[34]}$ presented a grain boundary pinning model (Zener model) ${ }^{[35]}$. This model considers a grain boundary mobility hindering when the driving force for grain growth is balanced with the counterforce exerted by the secondary phase distribution within the composite ${ }^{[36]}$. However, it has been extensively demonstrated that the general case considers certain features that are far from the real conditions and thus, fail to effectively predict the resulting mean grain size. A clear example of this is thoroughly discussed by Manohar et al. in their review of the evolution of the Zener model throughout the past years ${ }^{[35]}$. It is then clear, that a general descriptive model would not be realistic and therefore, each system should be modelled according to its particular set of processing parameters. 
Based on a generalization of the Zener model, Suarez et al. ${ }^{[21]}$ were further able to adapt it for the prediction of the mean grain size of a nickel metal matrix composite reinforced with different volume fractions of MWCNT by considering a descriptive shape factor of the CNT. This approach fits perfectly with the experimental data but, as already stated, is specifically linked to the conditions described in the report.

Considering this as a starting point, the present study is focused on the adaptation of a Zener model to predict the final obtained mean grain size as a function of the amount of the three types of CNP studied. As information regarding hybridization state and particle morphology of carbon nanoparticles is considered in this study, it provides valuable information for CNPreinforced metal matrix composites to a more general extent.

\section{Materials and methods}

\subsection{Production}

Nickel matrix composites reinforced with multiwall carbon nanotubes (Baytubes C150P, purity $>95 \%$, individual particle diameter of 5-20 nm), nanodiamonds (NaBond Technologies Co., purity $>98 \%$, individual particle diameter 4-8 nm) or onion-like carbon (obtained from nanodiamonds) are analysed. To synthesize OLC, the nanodiamond powder is annealed in graphite crucibles in furnace with tungsten heaters (Model: 1100-3580-W1, Thermal Technology Inc.) under vacuum $\left(1 \times 10^{-5}\right.$ mbar $)$ using a heating and cooling rate of $15^{\circ} \mathrm{C} / \mathrm{min}$ and a maximum temperature of $1750{ }^{\circ} \mathrm{C}$ (hold for $3 \mathrm{~h}$ ). The particle agglomerates are dispersed in ethylene glycol using a homogenizer (WiseTis, Witeg) and an ultrasonic bath. The processing steps are described elsewhere ${ }^{[23]}$. The obtained dispersions are finally used as precursors to manufacture nickel matrix composites with Ni dendritic powder (Alfa Aesar, -325 mesh) with a particle volume fractions of $0.5,1,3,6.5$, and 10 vol. $\%$. A hot uniaxial press (axial pressure of $264 \mathrm{MPa})$ under vacuum $\left(2 \times 10^{-6} \mathrm{mbar}\right)$ is used at $700{ }^{\circ} \mathrm{C}$ for $2.5 \mathrm{~h}$ to densify the powder 
mixture ${ }^{[23]}$. After densification, an additional annealing step $\left(550^{\circ} \mathrm{C}\right.$ for $6 \mathrm{~h}$ under vacuum at $2 \times 10^{-6}$ mbar) was added in order to allow for an extended grain growth.

\subsection{Characterization}

After the production of the composites, the samples are polished up to $1 \mu \mathrm{m}$ polishing suspension after which an oxide polishing suspension (OPS) is used as final preparation step. The samples are then characterized in a dual beam workstation FE-SEM/FIB Helios NanoLab $600^{\mathrm{TM}}$ (FEI). The mean grain size of the matrix after sintering is measured by electron backscattered diffraction (EBSD), with an EDAX TSL ${ }^{\mathrm{TM}}$ detector attached to the electron microscope. The scanned areas are at least of $(250 \times 250) \mu \mathrm{m}$ applying an accelerating voltage of $20 \mathrm{kV}$, a current of $22 \mathrm{nA}$, and a step size of $0.3 \mu \mathrm{m}$. For the evaluation, a grain was defined as at least two adjacent points with a misorientation lower than $5^{\circ}$, beyond which a grain boundary is set. In order to have reliable and representative statistics, at least 10000 grains are considered for each nanoparticle concentration. The acquired raw data is further post-processed using confidence index (CI) standardization, followed by the removal of points with CI below 0.09. All grains intersecting the scan window were removed from the analysis.

Furthermore, SEM was used to record micrographs of all different composites at three independent spots (always same magnification of 500 times with a resolution of 1024x884) in order to correlate the mean grain size with the mean agglomerate diameter. For analysis, the three micrographs were each divided in four subsections, which were binarised and segmented using the a4i (Archive4Images) resulting in 12 sub-segments for each particle type and concentration. This allows determining the area of each individual agglomerate within the subsegment. From the area, an equivalent agglomerate diameter was calculated assuming the agglomerates to be fairly round. The agglomerate diameter distribution for all segments was then classified into 20 equidistant sections to allow for a graphical illustration of the distribution. 
Also, the analysed areas were mapped by energy dispersive X-ray spectroscopy (EDX) in order to correlate areas with high carbon content to the SEM micrographs. An accelerating voltage of $5 \mathrm{kV}$ and a current of $22 \mathrm{nA}$ has been used.

\section{Results and discussion}

\subsection{Microstructure and particle distribution}

The acquired grain size data has to be fitted in order to calculate the mean grain size and the standard deviation. All samples showed a log-normal type of grain size distribution. Table 1 shows the mean grain size values for each concentration (area weighed).

Table 1. Mean grain sizes and their respective standard deviation for all the studied nanoparticles and volume fractions. The values were obtained from EBSD scans. The saturation value for each type of nanoparticle is highlighted in grey.

\begin{tabular}{|c|c|c|c|c|c|c|}
\hline \multirow{2}{*}{$\begin{array}{c}\text { Vol. } \\
\text { fraction } \\
/ \%\end{array}$} & \multicolumn{2}{|c|}{ CNT } & \multicolumn{2}{c|}{ OLC } & \multicolumn{2}{c|}{ nD } \\
\cline { 2 - 7 } & $\begin{array}{c}\langle\mathrm{d}\rangle_{\text {Area }} \\
/ \mu \mathrm{m}\end{array}$ & $\pm \sigma_{\text {Area }} / \mu \mathrm{m}$ & $\begin{array}{c}\langle\mathrm{d}\rangle_{\text {Area }} \\
/ \mu \mathrm{m}\end{array}$ & $\begin{array}{c} \pm \sigma_{\text {Area }} \\
/ \mu \mathrm{m}\end{array}$ & $\begin{array}{c}\langle\mathrm{d}\rangle_{\text {Area }} \\
/ \mu \mathrm{m}\end{array}$ & $\begin{array}{c} \pm \sigma_{\text {Area }} \\
/ \mu \mathrm{m}\end{array}$ \\
\hline 0 & 19.57 & 15.61 & 19.57 & 15.61 & 19.57 & 15.61 \\
\hline 0.5 & 8.20 & 9.52 & 5.58 & 7.75 & 7.86 & 7.77 \\
\hline 1 & 4.66 & 2.39 & 3.38 & 1.58 & 3.26 & 1.64 \\
\hline 3 & 4.01 & 2.31 & 3.70 & 1.84 & 3.55 & 1.78 \\
\hline 6.5 & 4.23 & 2.17 & 2.74 & 1.53 & 2.78 & 1.55 \\
\hline 10 & 4.50 & 2.53 & 2.95 & 1.49 & 2.32 & 1.39 \\
\hline
\end{tabular}

In all samples there is a clear downward trend to a refinement saturation value, beyond which the mean grain size starts to increase. Reinert et al. ${ }^{[23]}$ demonstrated that differences in the agglomerate sizes and the distribution of the three used CNP can be expected, with $\mathrm{nD}$ showing the lowest and CNT showing the highest tendency to form large agglomerates. Interestingly, this trend can be correlated to the here obtained saturation values for the grain refinement ${ }^{[23]}$. For smaller agglomerate sizes (nD-containing samples), a better distribution of the nanoparticles is expected, thus enhancing their grain boundary pinning efficiency up to larger volume fractions. Samples that present a larger agglomerate size (CNT-containing samples), do not efficiently act on the moving boundaries during sintering. This has two main reasons: First, larger agglomerates mean that there is a lower amount of individually distributed 
particles at the grain boundaries, and second, large agglomerates usually present a poor interfacial cohesion to the matrix, which translates into an inefficient boundary drag and load transfer ${ }^{[21]}$.

Due to the nature of the powder blending and manufacturing process, all types of particles are placed at grain boundaries ${ }^{[37]}$. It is therefore rational to assume that all the refinement process would take place only if a proper interfacial contact between reinforcement and matrix is provided. Furthermore, it is well known that Ni does not form any type of carbide phases when combined with $\mathrm{C}$ (under normal conditions) ${ }^{[38]}$. The Ni-C phase diagram shows a single metastable carbide $\left(\mathrm{Ni}_{3} \mathrm{C}\right)$, which is formed only under certain non-equilibrium conditions ${ }^{[39]}$. On one hand, the formation of this phase would be certainly beneficial for the interaction of the grain boundaries with the reinforcement by providing a seamless interfacial contact. However, it would be detrimental from the perspective of retaining the outstanding physical properties of the nanoparticles. Based on the resulting grain size values reported in Table 1, it is valid to state that the particles present a very good interfacial contact to the matrix without chemically reacting with it, thus highlighting the remarkable efficiency of the manufacturing process. This has been previously analysed and discussed specifically for the Ni-CNT system ${ }^{[40]}$, but can be straightforwardly transferred to both, the Ni-OLC and Ni-nD systems. 

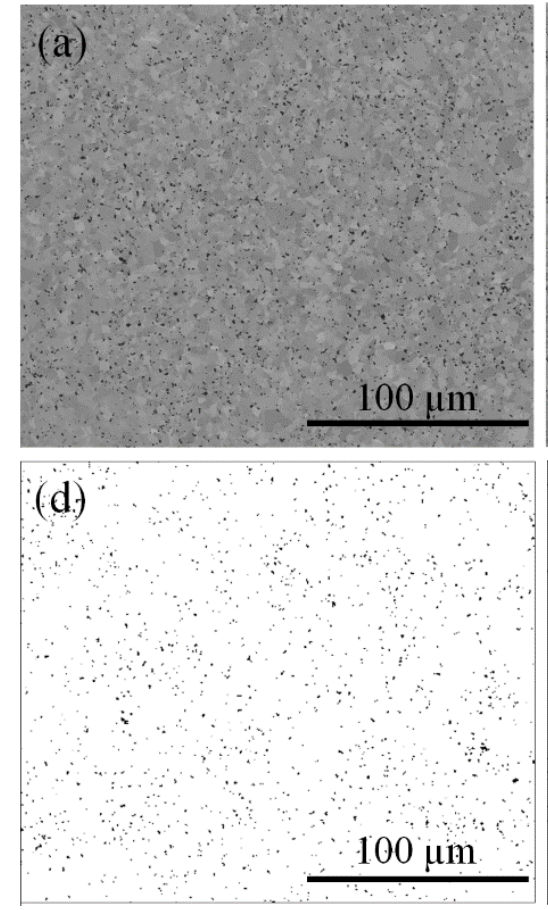

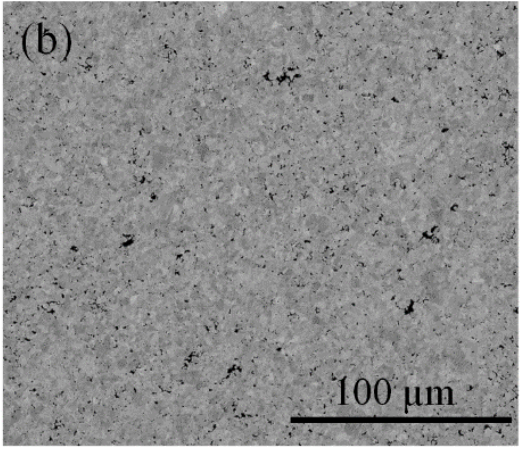

$(\mathrm{e})$

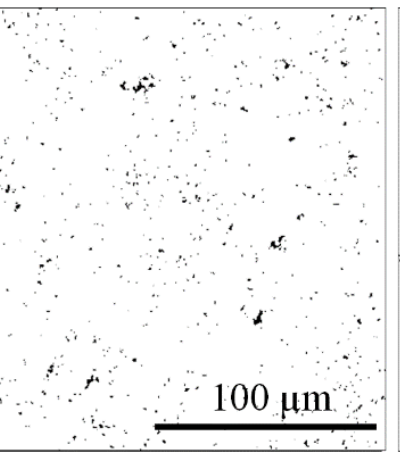

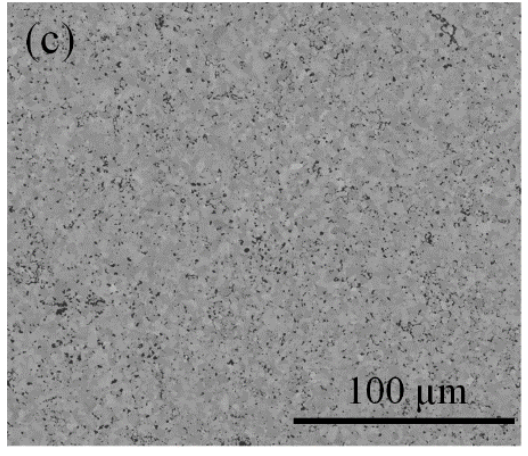

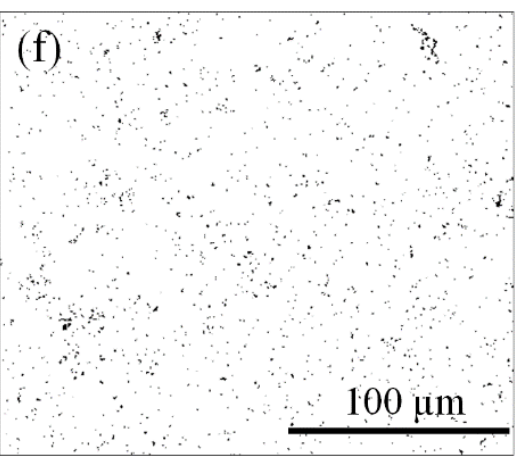

Figure 1 - Backscattered electron micrographs of the Ni-matrix composites with 3 vol.\% reinforcement fraction. of (a) CNT, (b) OLC and, (c) $n D$. The dark black regions are the different particle agglomerates. This is proven by EDX-mappings of the same areas for $(d)$ $C N T$, (e) $O L C$ and $(f) n D$. The black regions in these EDX-maps show areas with elementary carbon content, which correlates perfectly with the black areas of the backscattered electron micrographs.

Typical microstructures for the different reinforcement types are shown in Fig. 1. Particularly, the shown scanning electron micrographs (a-c) belong to the 3 vol.\% samples, where the black regions are the particle agglomerates. All show a homogeneous distribution and the particle agglomerates are placed at the grain boundaries. In order to prove that the black areas are CNPs, EDX-maps have been added below the corresponding SEM-micrograph. The Black areas in these EDX-maps represent the existence of elementary carbon. Thus, it can be seen, that the elementary carbon distribution correlates perfectly with the black areas from the SEM-micrographs. 
Fig. 2 shows the agglomerate size distribution histograms for each concentration.

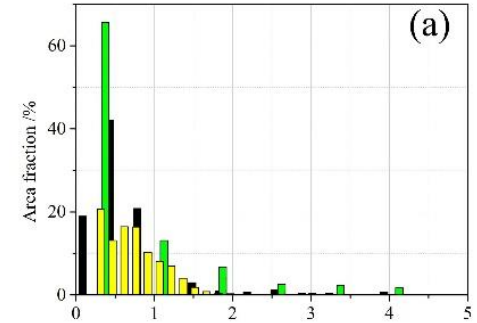

Equivalent mean diameter $\langle\mathrm{d}\rangle_{\mathrm{Aren}} / \mu \mathrm{m}$

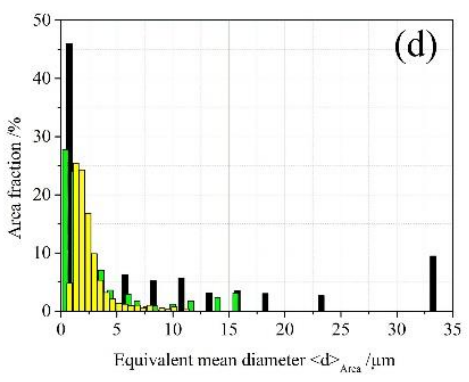

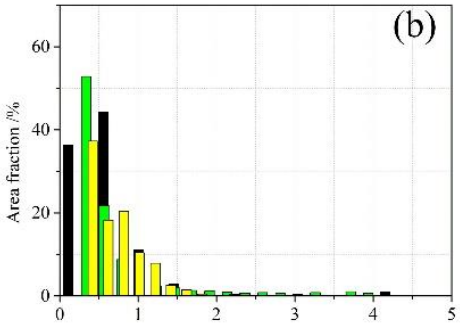

Equivalent mean diameter $<\mathrm{d}>/$ Anea $/ \mu \mathrm{m}$

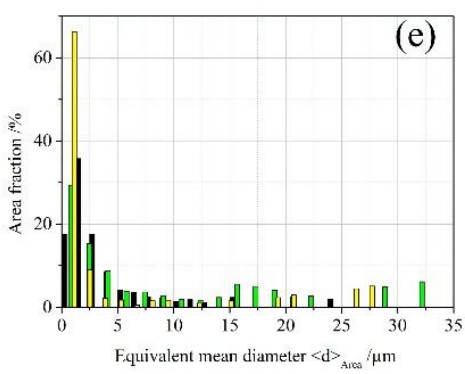

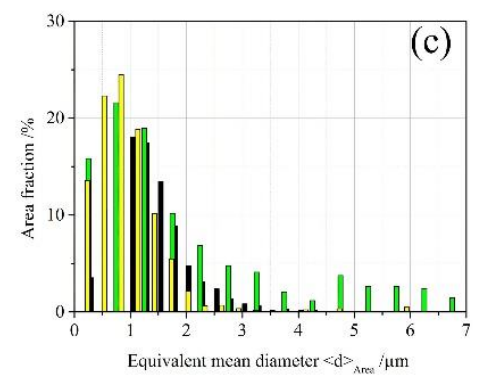

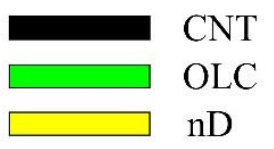

Figure 2 - Equivalent mean agglomerate diameter histograms of (a) 0.5 vol.\%, (b) 1 vol.\%, (c) 3 vol. $\%$, (d) 6.5 vol. $\%$ and, (e) 10 vol. $\%$.

It can be observed that in all cases, the $\mathrm{nD}$ samples show a narrower size distribution compared to those of CNT and OLC. This indicates a close to monomodal size distribution and is related to the weak agglomeration activity of $\mathrm{nD}$, which is traced back mainly to their hybridization ${ }^{[23]}$. CNT and OLC show a widespread, multimodal agglomerate size distribution. This is also related to their $\mathrm{sp}^{2}$ hybridization, in which the electron delocalization of the $\mathrm{p}$ orbitals favours their interaction and subsequent agglomeration due to van der Waals interactions.

The histograms show a correlation, to a certain extent, to the saturation behaviour identified in the mean grain size (Table 1). Thus, by increasing the particle fraction, the agglomerate size is also increased and the refinement efficiency is hindered. Specifically, the first 3 concentrations $(0.5,1$ and 3 vol.\%) show no significant differences between the agglomerate sizes (Fig. 2 a to c) as they are all much smaller than a maximum value of almost $7 \mu \mathrm{m}$. At 6.5 vol.\% the difference starts to be noticeable, showing a wider agglomerate distribution of the CNTs. Finally, the most critical case in which all samples show a spread distribution is for the 
highest evaluated concentration (i.e. 10 vol.\%). However, even for 10 vol.\%, $\mathrm{nD}$ still show by far the highest amount of small agglomerates, justifying their grain refinement effect for up to this concentration. When the mean grain sizes shown in Table 1 are contrasted to the pure Ni reference state (mean grain size of $19.5 \mu \mathrm{m}$ ), a maximum fivefold refinement for MWCNT, about 7-fold for OLC and about 8.5-fold for $\mathrm{nD}$ is accomplished. These resulting grain sizes correspond to 3 vol.\% of MWCNT, 6.5 vol.\% of OLC and 10 vol.\% of nD.

\subsection{Predictive microstructural modelling}

It has been already shown that the conventional theoretical models fail to predict the final mean grain size in these types of composites ${ }^{[21]}$. This is further supported by the amount of available models reported throughout the years, which start from the same original equation but render completely different outcomes ${ }^{[35]}$. Basically, the Zener model predicts the critical grain size at which the grain boundary driving and drag forces are balanced and no further growth is possible $^{[35,36]}$. There is extensive evidence that, in order to reliably model a biphasic system, each feature of the secondary phase must be considered. Specifically, in fibre-type reinforcements it is of utmost relevance to precisely know how the contact and relative orientation between the fibre and matrix might be. This is a non-trivial task, which can be straightforwardly overcome by assuming a random distribution of the fibre and considering certain morphological parameters that would ensure the consideration of the different contact possibilities. Specifically for the case of CNT reinforcements, Lahiri et al. ${ }^{[41]}$ proposed a very interesting variation of the Zener drag model, in which the specific surface area of the CNT is considered. For our particular case, we further simplify the modelling by considering the mean agglomerate size of CNP after their dispersion, which would fundamentally interact with the grain boundaries as a single body. By starting from the theoretical model proposed by Smith ${ }^{[42]}$, a simpler equation can be derived by combining two known factors (particle radius and volume fraction): 


$$
\bar{D}=\frac{K \cdot r}{f^{n}}
$$

In Equation 1, $D$ is the mean grain size, $r$ the mean radius of the particle agglomerates after dispersion, $f$ is the reinforcement volume fraction, and $K$ and $n$ are fitting constants that depend on the material system (matrix and reinforcement).

For the used dispersion process and parameters, it has been found that all three kinds of CNP behave in the same way, leading to a mean agglomerate radius of $40-70 \mathrm{~nm}^{[23]}$. Therefore, a mean agglomerate radius of $50 \mathrm{~nm}$ is assumed here as a first approximation. Furthermore, since the grain boundary pinning is more active at the onset of the grain growth (largest fraction of the energy is dissipated due to a larger grain boundary curvature ${ }^{[36]}$ ), the consideration of the initial agglomerate size is the most suitable way to approach the problem.

It has to be kept in mind, that the reagglomeration behavior of the different CNP differs, which will result in different final agglomerate sizes in the sintered composite as shown before in this report. However, as already mentioned, by assuming that all reinforcement types behave the same (different NP), it is reasonable to assume that the fundamental role in pinning is played by agglomerates and not individual NP. Additionally, no interfacial phases are expected to be formed. Under these circumstances, the equation can be expressed as follows:

$$
\bar{D}=\frac{0.99 \pm 0.07}{f^{0.4}}
$$

A n-coefficient of 0.4 can be found for systems where the secondary particles are placed at the grain boundaries ${ }^{[43]}$, which is true in the case of the used composites ${ }^{[22,23]}$. 


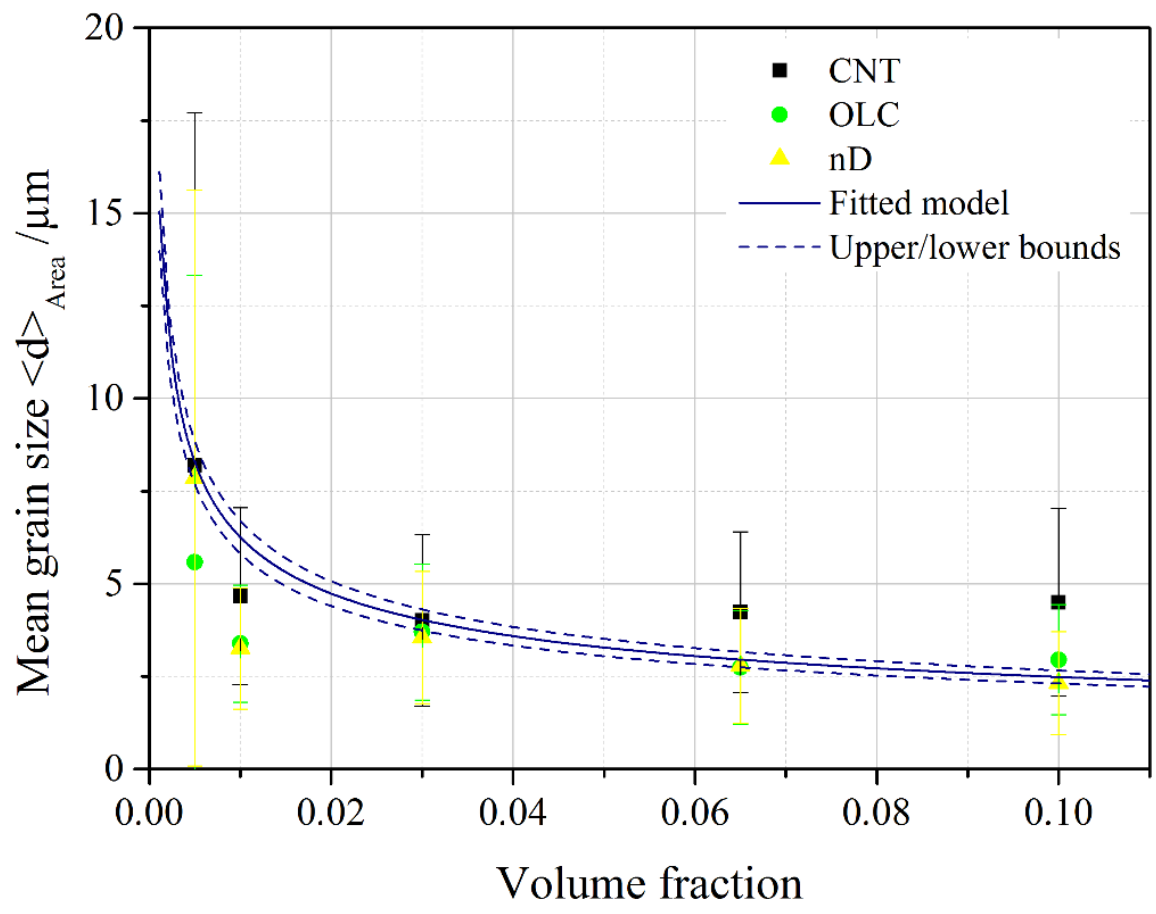

Figure 3 - mean grain size for all different studied systems as a function of the CNP volume fraction. The blue solid curve represents the empirical Zener boundary pinning model and the dashed blue lines show its upper and lower bounds as analytically shown in Equation 2.

In general, the model is in good agreement to all the experimental data obtained for $\mathrm{nD}$ and OLC, except for 1 vol.\%, which might be related to the low concentration and therefore an inhomogeneous distribution of the reinforcement phase (Fig. 3). However, the real mean grain size for CNT is twice as high as predicted by the model for concentrations higher than 3 vol.\%. This can be perfectly correlated to the CNT's saturation value for the grain refinement, as shown in Table 1. The CNT show a higher tendency to form agglomerates due to the high aspect ratio and $\mathrm{sp}^{2}$-hybridization, leading to a saturation value of the volume concentration lower than for OLC and $\mathrm{nD}^{[23]}$. The larger agglomerate sizes lead to a less pronounced grain pinning effect and therefore, the model is no longer valid for those concentrations.

Summarizing, it is observed that OLC and $\mathrm{nD}$ retain their agglomerate size at higher concentrations to a certain degree, resulting in a better distribution and thus a more efficient grain refinement. The CNT may show multiple interactions with the grain boundaries depending on how the grain bounaries come across the CNT. It is expected that the behaviour 
of the CNT-containing samples are governed by the randomness of the CNT distribution and their increased agglomerate size ${ }^{[44]}$. Finally it can be stated that the model proposed in this report is suitable for all three systems analysed for a wide concentration range, except for larger CNT concentrations where the model provides a fair approximation but with a certain deviancy resulting from the increased agglomeration activity.

\section{Concluding remarks}

After producing and measuring the mean grain size of CNT, OLC and $\mathrm{nD}$ reinforced nickel matrix composites, a model based on the Zener equation is successfully adapted to fit and predict the observed grain refinement for all three kinds of carbon nanoparticles. It is shown, that the model is perfectly valid as long as no saturation value of the grain refinement effect for the individual particle type is reached. However, the individual concentration at which a saturation value is reached differs and can be correlated to the mean agglomerate size distribution within the composites. This difference can be correlated to different reagglomeration behaviours due to different carbon hybridization states (sp2 vs. sp3) or particle geometries (0D vs. 1D) for the three kinds of carbon nanoparticles. In this respect, CNTs show the highest tendency to build larger agglomerates, thus reaching its grain refinement saturation value at lower concentrations than OLC and $\mathrm{nD}$. Compared to the unreinforced reference with a mean grain size of $19.5 \mu \mathrm{m}$, a maximum grain refinement of a factor of 5 for MWCNT, about 7 for OLC and about 8.5 for $\mathrm{nD}$ is achieved. These values correspond to 3 vol.\% of MWCNT, 6.5 vol.\% of OLC and 10 vol. \% of $\mathrm{nD}$. 


\section{References}

[1] Y. Gogotsi, V. Presser, Carbon Nanomaterials, 2nd Ed., CRC Press, Boca Raton FL, 2014.

[2] S.R. Bakshi, D. Lahiri, A. Agarwal, Carbon nanotube reinforced metal matrix composites - a review, Int. Mater. Rev. 55 (2010) 41-64.

[3] V.N. Mochalin, O. Shenderova, D. Ho, Y. Gogotsi, The properties and applications of nanodiamonds., Nat. Nanotechnol. 7 (2012) 11-23.

[4] J.Y. Hwang, B.K. Lim, J. Tiley, R. Banerjee, S.H. Hong, Interface analysis of ultra-high strength carbon nanotube/nickel composites processed by molecular level mixing, Carbon 57 (2013) 282-287.

[5] S.R. Bakshi, A. Agarwal, An analysis of the factors affecting strengthening in carbon nanotube reinforced aluminum composites, Carbon 49 (2011) 533-544.

[6] D. Nunes, M. Vilarigues, J.B. Correia, P. a. Carvalho, Nickel-carbon nanocomposites: Synthesis, structural changes and strengthening mechanisms, Acta Mater. 60 (2012) 737-747.

[7] D. Nunes, J.B. Correia, P.A. Carvalho, Nanodiamond dispersions in metallic matrices with different carbon affinity, Microsc. Microanal. 19 (2013) 2013.

[8] V. Livramento, J.B. Correia, N. Shohoji, E. Osawa, Nanodiamond as an effective reinforcing component for nano-copper, Diam. Relat. Mater. 16 (2007) 202-204.

[9] J.N. Boland, X.S. Li, Microstructural Characterisation and Wear Behaviour of Diamond Composite Materials, Materials (Basel). 3 (2010) 1390-1419.

[10] L. Kumari, T. Zhang, G. Du, W. Li, Q. Wang, A. Datye, et al., Thermal properties of CNT-Alumina nanocomposites, Compos. Sci. Technol. 68 (2008) 2178-2183.

[11] A. Agarwal, S.R. Bakshi, D. Lahiri, Carbon Nanotubes reinforced Metal Matrix Composites, 1st ed., CRC Press, Boca Raton, FL, USA, 2011.

[12] D. Nunes, J.B. Correia, P.A. Carvalho, Nanodiamond dispersions in nanostructured metals, Microsc. Microanal. 18 (2012) 73-74.

[13] Y. Li, B.X. Li, W.J. Zou, The Relationship between Nanocrystalline Structure and Frictional Properties of Nanodiamond/Ni Composite Coatings by Brush Plating, Appl. Mech. Mater. 80-81 (2011) 683-687.

[14] A. Hirata, M. Igarashi, T. Kaito, Study on solid lubricant properties of carbon onions produced by heat treatment of diamond clusters or particles, Tribol. Int. 37 (2004) 899905. 
[15] K. Miyoshi, K.W. Street, R.L. Vander Wal, R. Andrews, A. Sayir, Solid lubrication by multiwalled carbon nanotubes in air and in vacuum, Tribol. Lett. 19 (2005) 191-201.

[16] S. Iijima, Helical microtubules of graphitic carbon, Nature. 354 (1991) 56.

[17] H.W. Kroto, J.R. Heath, S.C. O`Brian, R.F. Curl, R.E. Smalley, C60: Buckminsterfullerene, Nature. 318 (1985) 162-163.

[18] C. Guiderdoni, E. Pavlenko, V. Turq, a. Weibel, P. Puech, C. Estournès, et al., The preparation of carbon nanotube (CNT)/copper composites and the effect of the number of CNT walls on their hardness, friction and wear properties, Carbon (2013).

[19] H. Dieringa, Properties of magnesium alloys reinforced with nanoparticles and carbon nanotubes: a review, J. Mater. Sci. 46 (2010) 289-306.

[20] H.J. Choi, J.H. Shin, D.H. Bae, Grain size effect on the strengthening behavior of aluminum-based composites containing multi-walled carbon nanotubes, Compos. Sci. Technol. 71 (2011) 1699-1705.

[21] S. Suarez, F. Lasserre, F. Mücklich, Mechanical properties of MWNT/Ni bulk composites: Influence of the microstructural refinement on the hardness, Mater. Sci. Eng. A. 587 (2013) 381-386.

[22] S. Suarez, E. Ramos-Moore, B. Lechthaler, F. Mücklich, S. Suárez, Grain growth analysis of multiwalled carbon nanotube bulk Ni composites, Carbon 70 (2014) 173178.

[23] L. Reinert, M. Zeiger, S. Suárez, V. Presser, F. Mücklich, Dispersion analysis of carbon nanotubes, carbon onions, and nanodiamonds for their application as reinforcement phase in nickel metal matrix composites, RSC Adv. 5 (2015) 95149-95159.

[24] V. Mochalin, S. Osswald, Y. Gogotsi, Contribution of Functional Groups to the Raman Spectrum of Nanodiamond Powders, Chem. Mater. 21 (2009) 273-279.

[25] A. Sanaty-Zadeh, Comparison between current models for the strength of particulatereinforced metal matrix nanocomposites with emphasis on consideration of Hall-Petch effect, Mater. Sci. Eng. A. 531 (2012) 112-118.

[26] S.C. Tjong, Recent progress in the development and properties of novel metal matrix nanocomposites reinforced with carbon nanotubes and graphene nanosheets, Mater. Sci. Eng. R Reports. 74 (2013) 281-350.

[27] S.D. Bergin, Z. Sun, D. Rickard, P. V Streich, J.P. Hamilton, J.N. Coleman, Multicomponent solubility parameters for single-walled carbon nanotube-solvent mixtures., ACS Nano. 3 (2009) 2340-50.

[28] M.R.S. Castro, Alternative Conductive Coatings Based on Multi-Walled Carbon 
Nanotubes, Ph.D. Thesis, Saarland University, 2007.

[29] A. Pentecost, S. Gour, V. Mochalin, I. Knoke, Y. Gogotsi, Deaggregation of nanodiamond powders using salt- and sugar-assisted milling., ACS Appl. Mater. Interfaces. 2 (2010) 3289-94.

[30] A. Krüger, F. Kataoka, M. Ozawa, T. Fujino, Y. Suzuki, A. E. Aleksenskii, et al., Unusually tight aggregation in detonation nanodiamond: Identification and disintegration, Carbon 43 (2005) 1722-1730.

[31] M. Zeiger, N. Jäckel, M. Aslan, D. Weingarth, V. Presser, Understanding structure and porosity of nanodiamond-derived carbon onions, Carbon 84 (2015) 584-598.

[32] K. Bogdanov, A. Fedorov, V. Osipov, T. Enoki, K. Takai, T. Hayashi, et al., Annealinginduced structural changes of carbon onions: High-resolution transmission electron microscopy and Raman studies, Carbon 73 (2014) 78-86.

[33] O.O. Mykhaylyk, Y.M. Solonin, D.N. Batchelder, R. Brydson, Transformation of nanodiamond into carbon onions: A comparative study by high-resolution transmission electron microscopy, electron energy-loss spectroscopy, x-ray diffraction, small-angle xray scattering, and ultraviolet Raman spectroscopy, J. Appl. Phys. 97 (2005) 074302.

[34] C.S. Smith, Grains, phases, and interphases: an interpretation of microstructure, Trans. Met. Soc. AIME. 175 (1948) 15-51.

[35] P.A.P. Manohar, M. Ferry, T. Chandra, Five decades of the Zener equation, ISIJ Int. 38 (1998) 913-924.

[36] D.A. Porter, K.E. Easterling, Phase Transformations in Metals and Alloys, 2nd ed., Chapman \& Hall, London, UK, 1992.

[37] P. Rossi, S. Suarez, F. Soldera, F. Mücklich, Quantitative Assessment of the Reinforcement Distribution Homogeneity in CNT/Metal Composites, Adv. Eng. Mater. 17 (2014) 1017-1021.

[38] J.Y. Hwang, A.R.P. Singh, M. Chaudhari, J. Tiley, Y. Zhu, J. Du, et al., Templated Growth of Hexagonal Nickel Carbide Nanocrystals on Vertically Aligned Carbon Nanotubes, J. Phys. Chem. C. 114 (2010) 10424-10429.

[39] M. Singleton, P. Nash, The C-Ni (Carbon-Nickel) system, Bull. Alloy Phase Diagrams. 10 (1989) 121-126.

[40] S. Suarez, F. Lasserre, O. Prat, F. Mücklich, Processing and interfacial reaction evaluation in MWCNT/Ni composites, Phys. Status Solidi. 211 (2014) 1555-1561.

[41] D. Lahiri, V. Singh, A.K. Keshri, S. Seal, A. Agarwal, Carbon nanotube toughened hydroxyapatite by spark plasma sintering: Microstructural evolution and multiscale 
tribological properties, Carbon 48 (2010) 3103-3120.

[42] C. Smith, Introduction to grains, phases, and interfaces - an interpretation of microstructure, Trans. AIME. 175 (1948) 15-51.

[43] G. Muralidharan, R.G. Thompson, Effect of second phase precipitation on limiting grain growth in alloy 718, Scr. Mater. 36 (1997) 755-761.

[44] N. Sun, B. Patterson, J. Suni, H. Weiland, L. Allard, Characterization of particle pinning potential, Acta Mater. 54 (2006) 4091-4099. 